\title{
Análise de Modelos Epidemiológicos para a COVID-19: Um Estudo de Casos de Belo Horizonte-MG durante a Pandemia de 2020
}

\author{
Gabriel A. D. Miranda ${ }^{1}$, Gustavo Y. S. Azevedo ${ }^{1}$, Matheus S. Garofolo ${ }^{1}$, \\ Lillia S. B. Silva ${ }^{2}$, Carlos A. Silva ${ }^{1}$ \\ ${ }^{1}$ Instituto Federal de Minas Gerais campus Sabará \\ Caixa Postal 34.590-390 - Sabará - MG - Brasil \\ ${ }^{2}$ Secretaria Municipal de Educação - Prefeitura Municipal de Sabará \\ Sabará, MG - Brasil
}

\{gabriel.alan32, gustavo.yallen, matheussaliba,lilliabarsante\}@gmail.com

carlos.silva@ifmg.edu.br

\begin{abstract}
In this paper, the epidemiological models SEIR and SIQR were used to evaluate the evolution of COVID-19 in the city of Belo Horizonte in Minas Gerais, Brazil, between the months of April to December 2020. The epidemiological models were solved using the of Runge-Kutta 4th-order, being implemented in Octave language. The simulation of data by the proposed models came close to the real evolution of COVID-19 in the region addressed, making these models good tools to improve the understanding of the dynamics of disease transmission.
\end{abstract}

Resumo. Neste trabalho foram utilizados os modelos epidemiológicos SEIR e SIQR para avaliar a evolução da COVID-19 no município de Belo Horizonte em Minas Gerais, Brasil, entre os meses de abril a dezembro de 2020. Os modelos epidemiológicos foram resolvidos usando o método numérico de Runge-Kutta de $4^{a}$ ordem, sendo implementados em linguagem Octave. A simulação dos dados pelos modelos propostos se aproximou da evolução real da COVID-19 na região abordada, tornando estes modelos boas ferramentas para aprimorar a compreensão da dinâmica de transmissão da doença.

\section{Introdução}

Em dezembro de 2019, a cidade de Wuhan na China tornou-se o epicentro de transmissão de um novo coronavírus (SARS- CoV-2) que em fevereiro de 2020 foi designado como COVID-19 pela Organização Mundial da Saúde (OMS) [Wu et al. 2020, Phelan et al. 2020]. A transmissão do coronavírus costuma ocorrer pelo ar em contato com secreções contaminadas, como gotículas de saliva, espirro, tosse e catarro ou no contato pessoal, como toque ou aperto de mão, e com objetos ou superfícies contaminadas, seguido de contato com a boca, nariz ou olhos [FCV 2020]. Os sintomas da COVID-19 podem variar de um resfriado até uma pneumonia severa. Sendo os sintomas mais comuns a tosse, febre, coriza, dor de garganta, dificuldade para respirar, perda de olfato, alteração do paladar, distúrbios gastrintestinais, cansaço, diminuição do apetite e dispnéia [MS 2020]. 
A COVID-19 espalhou-se rapidamente pelo mundo. Segundo a [OMS 2020], no ano de 2020 foram registrados mais de 82,3 milhões de casos confirmados globalmente. No Brasil, o primeiro caso confirmado de COVID-19 ocorreu em fevereiro de 2020 [Oliveira and Ortiz 2020] e somatizou até dezembro de 2020 mais de 7,6 milhões de casos registrados, sendo o terceiro país com mais casos confirmados do mundo [OMS 2020]. A região geográfica foco deste trabalho é a cidade de Belo Horizonte em Minas Gerais, a qual teve o primeiro caso registrado no dia 16 de março, totalizando até dezembro de 2020 mais de 63 mil casos.

Diversos estudos no mundo inteiro começaram a ser realizados com o objetivo de compreender e mapear o desenvolvimento da doença ou outras medidas que contribuam para a contenção e controle de sua transmissão [Buske et al. 2020, Manrique Abril et al. 2020]. De acordo com [Rocha 2012], a Epidemiologia é a ciência que estuda padrões da ocorrência de doenças em populações humanas e diferentes parâmetros que interferem na propagação de doenças e meios necessários à sua prevenção e controle. O primeiro modelo epidemiológico para estudar a disseminação de uma doença infecciosa na população foi proposto por [Kermark and Mckendrick 1927] denominado Modelo SIR (Suscetível - Infectado - Removido). Com o tempo, foram surgindo novos modelos derivados do SIR que utilizam mais parâmetros para otimizar os resultados, dentre eles o SEIR (Suscetível - Exposto - Infectado - Removido/Recuperado) e o SIQR (Suscetível - Infectado - Quarentena - Removido/Recuperado).

O presente trabalho propõe modelar os dados oficiais da COVID-19 registrados em Belo Horizonte utilizando os modelos SEIR e SIQR através do método numérico de Runge-kutta de $4^{a}$ ordem, dentre os meses de abril a dezembro de 2020. O objetivo foi comparar a evolução de ambos os modelos de acordo com a evolução da epidemia na cidade. Foram utilizados os dados oficiais fornecidos pela Secretaria Municipal de Saúde de Belo Horizonte [PBH 2020], bem como estimativas atuais do parâmetro de transmissão da doença dos modelos, tendo como base os dados globais da doença.

Nas seções 2 e 3 serão apresentados, respectivamente, os modelos epidemiológicos SEIR e SIQR e suas modelagens. A discussão dos resultados das simulações computacionais utilizando os modelos propostos, bem como uma comparação entre eles estará presente na seção 4. A conclusão e as considerações finais são apresentadas na seção 5 .

\section{Modelo epidemiológico SEIR}

O modelo epidemiológico SEIR separa a população em quatro compartimentos que refletem o estado dos indivíduos em relação ao desenvolvimento da doença em cada unidade de tempo $(t)$, sendo eles: indivíduos que estão suscetíveis a contrair a doença $S(t)$; indivíduos suscetíveis que ficaram expostos a indivíduos infectados $E(t)$; indivíduos que contraíram a doença e podem transmiti-la à indivíduos suscetíveis $I(t)$; e indivíduos que se infectaram e se recuperaram, adquirindo imunidade da doença $R(t)$ [Rocha 2012, Alves 2019, Hamzah et al. 2020].

O sistema de equações diferenciais ordinárias que representa o modelo SEIR sem dinâmica vital é dado por: 


$$
\begin{aligned}
d S(t) / d t & =-(\beta / N) S(t) I(t) \\
d E(t) / d t & =(\beta / N) S(t) I(t)-\sigma E(t) \\
d I(t) / d t & =\sigma E(t)-\gamma I(t) \\
d R(t) / d t & =\gamma I(t)
\end{aligned}
$$

em que $\beta$ representa a taxa de infecção, $\gamma$ representa a taxa de recuperação, $\sigma$ representa o período latente do patógeno e a constante $N$ denota a população total da região em estudo, sendo $N=S+E+I+R$. As populações e os parâmetros epidemiológicos são não negativos.

No modelo (1), percebe-se que cada equação descreve a entrada e a saída do indivíduo de um compartimento específico, sendo que as entradas são representadas com as adições e as saídas são representadas com as subtrações.

Um parâmetro importante em qualquer epidemia é o número de reprodução basal $R_{0}$ que mensura o número médio de novos infectados que um único indivíduo infectado consegue contaminar em uma população totalmente suscetível. Se $R_{0}>1$ implicará no crescimento exponencial do número de novas infecções e persistência da doença. No caso em que $R_{0}<1$ significa que o número de novos infectados irá decrescer e a doença tenderá à erradicação [Cohen 1992, Van den Driessche and Watmough 2002].

O $R_{0}$ do modelo (1) foi calculado analiticamente pelo raio espectral (maior autovalor em módulo) da matriz de próxima geração $G=\mathcal{V} \mathcal{W}^{-1}$, em que $\mathcal{V}$ e $\mathcal{W}$ são as matrizes jacobianas das funções de infecção $P$ e de transição $Z$ dos compartimentos $x=(E, I)$, avaliadas no ponto de equilíbrio livre de infecção da doença $(S, E, I, R)=(1,0,0,0)$ [Van den Driessche and Watmough 2002]:

$$
\begin{gathered}
P(x)=\left[\begin{array}{c}
(\beta / N) S I \\
0
\end{array}\right], Z(x)=\left[\begin{array}{c}
\sigma E \\
-\sigma E+\gamma I
\end{array}\right], \mathcal{V}=\left[\begin{array}{cc}
0 & \beta / N \\
0 & 0
\end{array}\right], \\
\mathcal{W}=\left[\begin{array}{cc}
\sigma & 0 \\
-\sigma & \gamma
\end{array}\right], \mathcal{W}^{-1}=\left[\begin{array}{cc}
1 / \sigma & 0 \\
1 / \gamma & 1 / \gamma
\end{array}\right] \text { e } G=\left[\begin{array}{cc}
(\beta / N \gamma) & (\beta / N \gamma) \\
0 & 0
\end{array}\right] .
\end{gathered}
$$

Desta forma, o número de reprodução basal no modelo SEIR é dado por

$$
R_{0}=\frac{\beta}{\gamma N}
$$

Outro parâmetro utilizado na epidemiologia é o número de reprodução efetivo $R T$, que tem definição similar à do $R_{0}$, com a diferença de que é uma métrica dinâmica e leva em consideração mudanças que podem afetar o valor do $R_{0}$ da doença, como o distanciamento social e o uso de máscaras [Fambrini and Gayer 2020].

\section{Modelo epidemiológico SIQR}

O modelo epidemiológico SIQR, semelhante ao modelo SEIR também separa a população em quatro classes que refletem o estado dos indivíduos em relação ao desenvolvimento da doença em cada unidade de tempo $t$, sendo elas: indivíduos que estão suscetíveis a contrair a doença $S(t)$; indivíduos que contraíram a doença e podem transmiti-la à indivíduos 
suscetíveis $I(t)$; indivíduos que contraíram a doença e se isolaram da comunidade $Q(t)$; e indivíduos que se infectaram e se recuperaram, adquirindo imunidade da doença $R(t)$ [Crokidakis 2020].

O sistema de equações diferenciais ordinárias que representa o modelo SIQR sem dinâmica vital é dado por:

$$
\begin{aligned}
d S(t) / d t & =-(\beta / N) S(t) I(t) \\
d I(t) / d t & =(\beta / N) S(t) I(t)-(\alpha+\eta) I(t) \\
d Q(t) / d t & =\eta I(t)-\gamma Q(t) \\
d R(t) / d t & =\gamma Q(t)+\alpha I(t),
\end{aligned}
$$

em que $\beta$ representa a taxa de infecção, $\alpha$ representa a taxa de recuperação de indivíduos infectados assintomáticos, $\eta$ representa a taxa de detecção de indivíduos infectados sintomáticos, $\gamma$ representa a taxa de recuperação de indivíduos em quarentena e a constante $N$ denota a população total da região em estudo, sendo $N=S+I+Q+R$. As populações e os parâmetros epidemiológicos são não negativos.

Assim como no modelo (1), percebe-se que cada equação do modelo (3) descreve a entrada e a saída do indivíduo de um compartimento específico, sendo que as entradas são representadas com as adições e as saídas são representadas com as subtrações.

O $R_{0}$ do modelo (3) foi calculado de forma análoga ao modelo (1) considerando os compartimentos $x=(I, Q)$ e o ponto de equilíbrio livre de infecção da doença $(S, I, Q, R)=(1,0,0,0)$ :

$$
\begin{gathered}
P(x)=\left[\begin{array}{c}
(\beta / N) S I \\
\eta I
\end{array}\right], Z(x)=\left[\begin{array}{c}
(\alpha+\eta) I \\
\gamma Q
\end{array}\right], \mathcal{V}=\left[\begin{array}{cc}
\beta / N & 0 \\
\eta & 0
\end{array}\right], \\
\mathcal{W}=\left[\begin{array}{cc}
(\alpha+\eta) & 0 \\
0 & \gamma
\end{array}\right], \mathcal{W}^{-1}=\left[\begin{array}{cc}
1 /(\alpha+\eta) & 0 \\
0 & 1 / \gamma
\end{array}\right] \text { e } G=\left[\begin{array}{cc}
\beta /[(\alpha+\eta) N] & 0 \\
\eta /(\alpha+\eta) & 0
\end{array}\right] .
\end{gathered}
$$

Desta forma, o número de reprodução basal no modelo SIQR é dado por

$$
R_{0}=\frac{\beta}{(\alpha+\eta) N}
$$

\section{Resultados e discussões}

Minas Gerais é o estado com a $2^{\mathrm{a}}$ menor taxa de testes para coronavírus por habitante no Brasil [G1 2020]. Sua capital, Belo Horizonte, registrou o primeiro caso de COVID19 em 16 de março de 2020, porém, somente a partir do dia 20 de abril de 2020 as [PBH 2020, SESMG 2020] começaram a divulgar boletins epidemiológicos diários. A base de dados utilizada neste trabalho é baseada nos boletins oficiais publicados, os quais não atualizam as informações nos finais de semana e feriados, sendo estas acrescentadas no dia útil seguinte, juntamente com os novos casos registrados do dia. Com isto, para validar o estudo comportamental dos modelos com os dados oficiais coletados, foi preenchida as lacunas via interpolação linear e adotado o sistema de média móvel (MM) de 7 dias, em que o tempo inicial foi a MM do dia 27/04/2020 e o tempo final foi a MM do dia $31 / 12 / 2020$, totalizando 249 dias. 
A Figura 1 representa o $R T$ utilizado neste trabalho, sendo este parâmetro fixo em cada semana. Estes valores foram baseados em [Figueiredo 2020] que utilizou o método de distribuição Gamma referente à COVID-19 na cidade de Belo Horizonte. A fim de diminuir o valor de $R T$ no horizonte de estudo, em diversos momentos foram implantadas ações governamentais por meio de decretos no qual suspendia as atividades comerciais não essenciais na cidade, como casas de festas, shoppings, academias, bares, escolas, dentre outros.

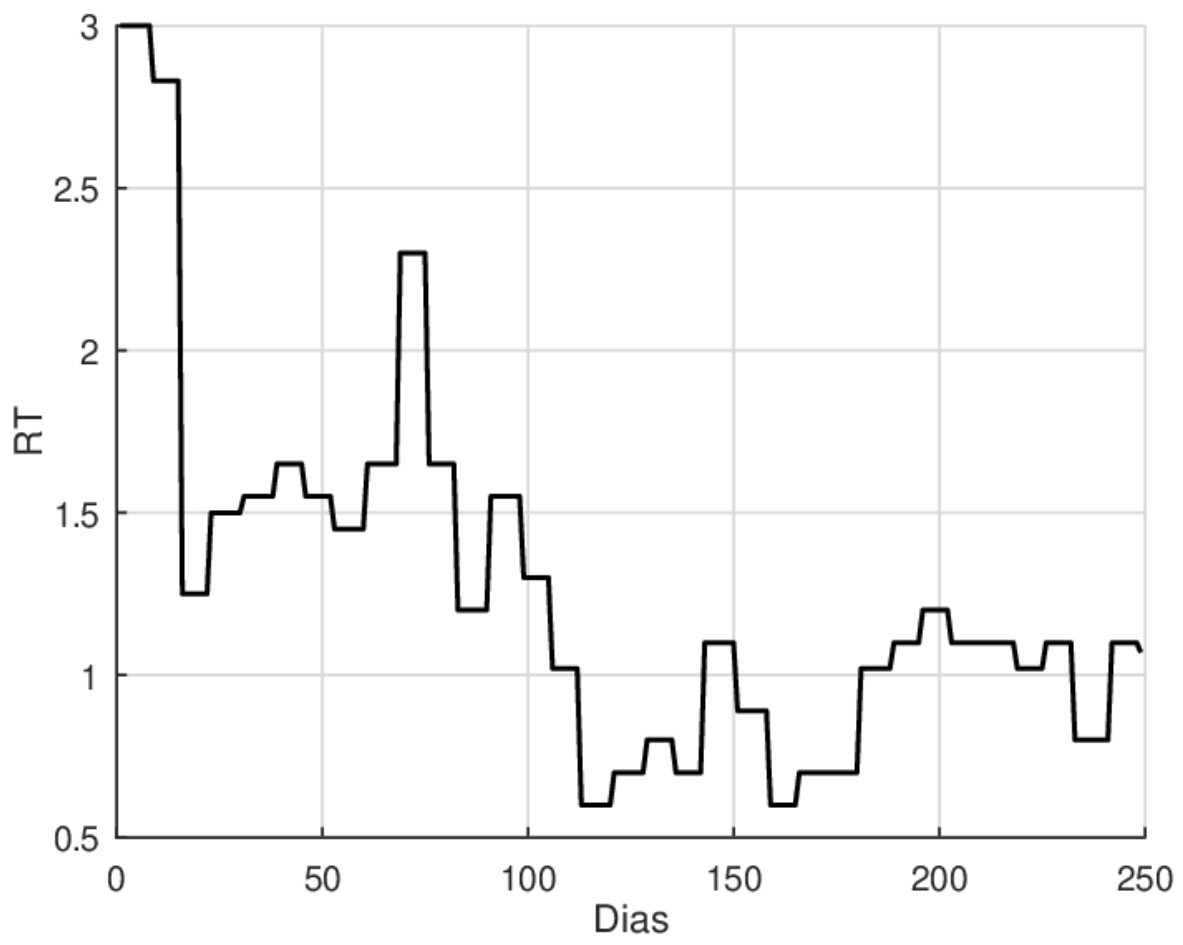

Figura 1. Número de reprodução efetivo da COVID-19 em Belo Horizonte de 27/04 a 31/12 de 2020. Fontes: [Figueiredo 2020] e [PBH 2020].

A taxa de infecção da doença foi obtida respectivamente pelas equações (2) e (4), em que foi aproximado o valor de $R_{0}$ pelo valor de $R T$. Foram consideradas para as condições iniciais do modelo SEIR os valores $I_{0}=\frac{21,43}{N}, R_{0}=0, E_{0}=0$ e $S_{0}=1-$ $R_{0}-I_{0}-E_{0}$, sendo respectivamente a fração de novos indivíduos infectados, recuperados, expostos e suscetíveis no primeiro dia da análise. No modelo SIQR foram consideradas para as condições iniciais os valores $I_{0}=\frac{21,43}{N}, R_{0}=0, Q_{0}=0$ e $S_{0}=1-R_{0}-I_{0}-E_{0}$ representando respectivamente a fração de novos indivíduos infectados, recuperados, em quarentena e suscetíveis.

Os parâmetros fixos adotados nas simulações dos modelos (1) e (3) foram descritos na Tabela 1. 
Tabela 1. Parâmetros adotados nos modelos (1) e (3).

\begin{tabular}{|c|c|c|c|}
\hline Símbolo & Valor & Unidade & Fonte \\
\hline$\sigma$ & $1 / 5$ & & {$[\mathrm{MS} 2020]$} \\
\hline$\gamma$ & $1 / 14$ & \multirow{2}{*}{$\operatorname{dias}^{-1}$} & \\
\cline { 1 - 2 } & $1 / 50$ & & {$[$ Crokidakis 2020] } \\
\hline$\alpha$ & $1 / 50$ & & {$[\mathrm{PBH} 2020]$} \\
\hline$N$ & 2501576 & - & - \\
\hline$t$ & 249 & - & \\
\hline
\end{tabular}

A Figura 2 apresenta o comportamento dos modelos SEIR e SIQR em relação à evolução do número de novos infectados, comparados com a média móvel dos dados registrados pelos boletins epidemiológicos. Apesar da simplicidade dos modelos, é possível observar um padrão no comportamento entre os dados simulados pelos modelos e os dados oficiais. Observe pela média móvel que o número de novos infectados cresce de forma exponencial, alcançando seu maior valor no dia 31/07/2020 $(t=96)$. O modelo SIQR chega no ápice no dia 15/08/2020 ( $t=111)$ e o SEIR alcança o pico no dia 16/08/2020 $(t=112)$. É válido mencionar, conforme [Li et al. 2020], que o número de casos da COVID-19 pode ser superior ao número de casos testados e confirmados.

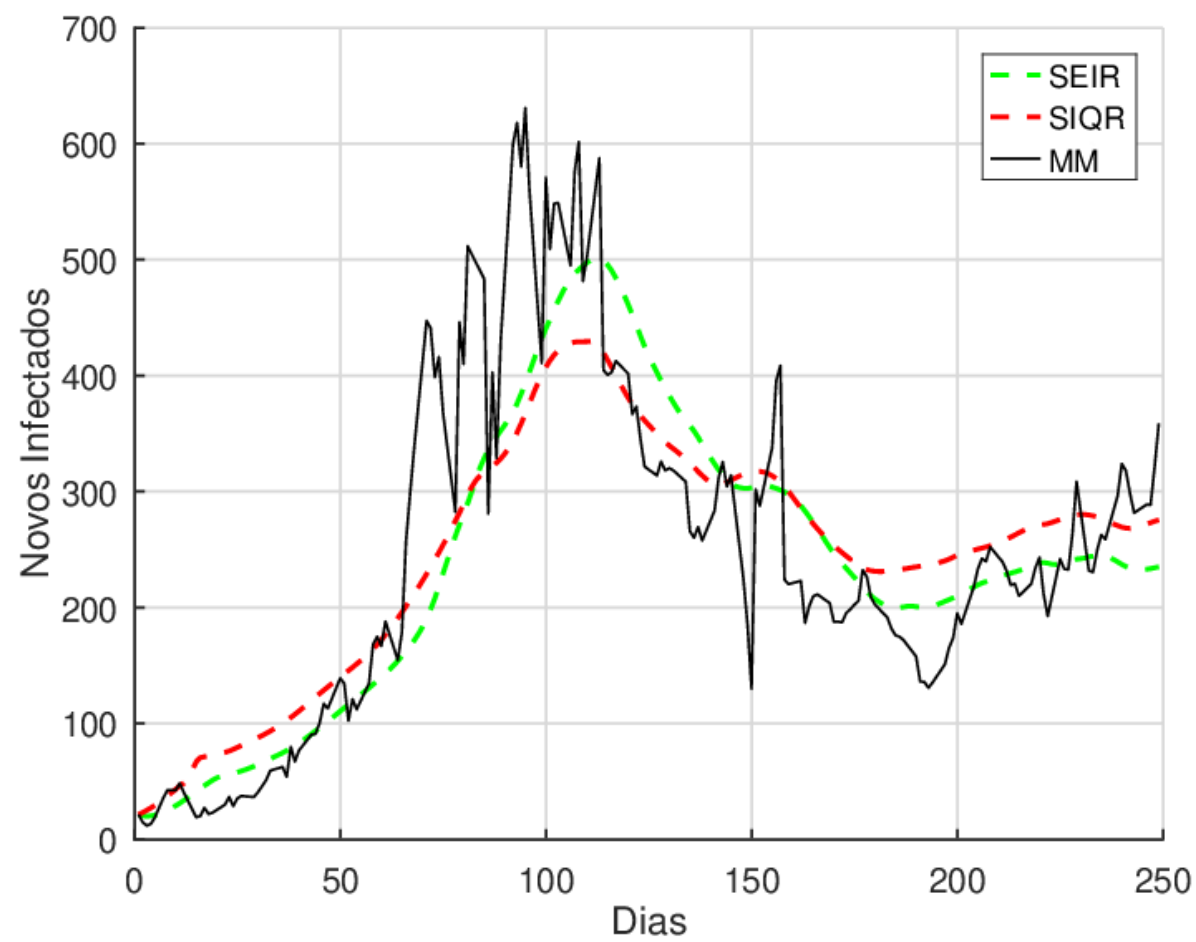

Figura 2. Comparativo dos modelos SEIR, SIQR e dados oficiais de novos casos de infectados por COVID-19 em Belo Horizonte no período de 27/04/20 a $31 / 12 / 20$. 


\section{Conclusão}

Neste trabalho foi proposto o estudo e comparação de dois modelos epidemiológicos, SEIR e SIQR, na dinâmica da COVID-19. Os resultados simulados foram comparados com os dados oficiais divulgados pela prefeitura de Belo Horizonte, juntamente com a Secretaria de Saúde do Estado de Minas Gerais. Foi possível perceber que os dados numéricos simulados, de ambos os modelos indicaram a tendência da dispersão dos dados oficiais para os parâmetros e período analisado.

A partir desta análise é possível afirmar que os modelos epidemiológicos, podem configurar-se como boas ferramentas de estratégia e simulação de epidemias, considerando a aproximação dos dados simulados pelos modelos ou indicação da tendência com a evolução real da epidemia. Desta forma é possível fazer estimativas da taxa de infecção, da taxa de isolamento de indivíduos da população, bem como do número de reprodução efetivo para traçar estratégias referentes aos resultados obtidos, auxiliando a tomada de decisão dos gestores públicos.

Ressalta-se que a cidade de Belo Horizonte ainda apresenta uma tendência de aumento de novas infecções diárias e que as políticas de isolamento e distanciamento social realmente funcionam e colaboram para o controle e diminuição do número de reprodução efetivo da COVID-19. Para trabalhos futuros seria de grande importância a abordagem do impacto das políticas de vacinação e isolamento social, bem como a análise de óbitos relacionados à doença.

\section{Referências}

Alves, G. A. (2019). A conta que fecha a reportagem: palco e bastidores em três casos de matemática aplicada. PhD thesis, Universidade de São Paulo.

Buske, D., Gonçalves, G. A., and de Quadros, R. S. (2020). A evolução epidêmica do COVID-19 - Modelo Sir. https://wp.ufpel.edu.br/fentransporte/ 2020/04/09/a-evolucao-epidemica-do-covid-19-modelo-sir/. Acesso: 17 dez. 2020.

Cohen, J. E. (1992). Infectious Diseases of Humans: Dynamics and Control. JAMA, 268(23):3381-3381.

Crokidakis, N. (2020). Covid-19 spreading in Rio de Janeiro, Brazil: Do the policies of social isolation really work? Chaos, Solitons \& Fractals, 136:109930.

Fambrini, F. and Gayer, V. (2020). Método computacional para determinação do parâmetro $R_{t}$ e sua relevância no controle da epidemia covid-19 no contexto da cidade de Bragança Paulista. Anais do WCF , 7:61-66.

FCV (2020). Fundação Cristiano Varela - Saiba tudo sobre o Coronavírus. http: // www.fcv.org.br/site/noticia/detalhe/1092. Acesso: 17 dez. 2020.

Figueiredo, F. (2020). Estimativas de $R(t)$ por estados do Brasil. https:// github.com/flaviovdf/covid19. Acesso: 17 dez. 2020.

G1 (2020). Portal de notícias globo. https://g1.globo.com/mg/minasgerais/noticia/2020/05/15/minas-gerais-e-o-estado-com-a2a-menor-taxa-de-testes-para-coronavirus-por-habitanteno-brasil.ghtml. Acesso: 17 dez. 2020. 
Hamzah, F. B., Lau, C., Nazri, H., Ligot, D., Lee, G., Tan, C., Shaib, M., Zaidon, U., Abdullah, A., Chung, M., Ong, C., Chew, P., and Salunga, R. (2020). Coronatracker: worldwide COVID-19 outbreak data analysis and prediction. Bull World Health Organ, 1:1-32.

Kermark, O. W. and Mckendrick, A. G. (1927). Contributions to the mathematical theory of epidemics. Part I. Proceedings of the Royal Society, 115(772):700-721.

Li, R., Pei, S., Chen, B., Song, Y., Zhang, T., Yang, W., and Shaman, J. (2020). Substantial undocumented infection facilitates the rapid dissemination of novel coronavirus (SARS-CoV-2). Science, 368(6490):489-493.

Manrique Abril, F., Agudelo Calderon, C. A., González-Chordá, V. M., Gutiérrez Lesmes, O. A., Tellez Piñerez, C. F., and Herrera-Amaya, G. M. (2020). Modelo SIR de la pandemia de COVID-19 en Colombia. Revista de Salud Pública, 22:1-9.

MS (2020). Ministério da Saúde - Coronavírus. https: // coronavirus.saude.gov.br/ e https://covid.saude.gov.br/. Acesso: 17 dez. 2020.

Oliveira, E. and Ortiz, B. (2020). Ministério da Saúde confirma primeiro caso de coronavírus no Brasil. https://gl.globo.com/ciencia-e-saude/noticia/ $2020 / 02 / 26 / m i n i s t e r i o-d a-s a u d e-f a l a-s o b r e-c a s o-p o s s i v e l-$ paciente-com-coronavirus.ghtml. Acesso: 29 dez. 2020.

OMS (2020). Organização Mundial de Saúde. https : / / covid19.who.int. Acesso: 17 dez. 2020.

PBH (2020). Prefeitura de Belo Horizonte. https://prefeitura.pbh.gov.br/ saude/coronavirus. Acesso: 28 jan. 2021.

Phelan, A. L., Katz, R., and Gostin, L. O. (2020). The novel coronavirus originating in Wuhan, China: challenges for global health governance. Jama, 323(8):709-710.

Rocha, D. I. C. (2012). Modelos matemáticos aplicados à epidemiologia. Master's thesis, Faculdade de Economia da Universidade do Porto.

SESMG (2020). Secretaria Estadual de Saúde de Minas Gerais. https:// coronavirus.saude.mg.gov.br. Acesso: 19 dez. 2020.

Van den Driessche, P. and Watmough, J. (2002). Reproduction numbers and sub-threshold endemic equilibria for compartmental models of disease transmission. Mathematical Biosciences, 180(1):29-48.

Wu, F., Zhao, S., Yu, B., Chen, Y.-M., Wang, W., Song, Z.-G., Hu, Y., Tao, Z.-W., Tian, J.-H., Pei, Y.-Y., Yuan, M.-L., Zhang, Y.-L., Dai, F.-H., Liu, Y., Wang, Q.-M., Zheng, J.-J., Xu, L., Holmes, E. C., and Zhang, Y.-Z. (2020). A new coronavirus associated with human respiratory disease in China. Nature, 579(7798):265-269. 